\title{
Energy Efficiency with QoS Control in Dynamic Optical Networks with SDN Enabled Integrated Control Plane
}

\author{
Jiayuan Wang ${ }^{\mathrm{a}}$, Xin Chen ${ }^{\mathrm{b}, *}$, Chris Phillips ${ }^{\mathrm{b}}$, Ying Yan ${ }^{\mathrm{a}}$ \\ ${ }^{a}$ DTU Fotonik, Oersted Plads, 2800 Kgs. Lyngby, Denmark \\ ${ }^{b}$ EECS, Queen Mary University of London, E1 4DW, UK
}

\begin{abstract}
The paper presents energy efficient routing algorithms based on a novel integrated control plane platform. The centralized control plane structure enables the use of flexible heuristic algorithms for route selection in optical networks. Differentiated routing for various traffic types is used in our previous work. The work presented in this paper further optimizes the energy performance in the whole network by utilizing a multi-objective evolutionary algorithm for route selection. The trade-off between energy optimization and QoS for high priority traffic is examined and results show an overall improvement in energy performance whist maintaining satisfactory QoS. Energy savings are obtained on the low priority traffic whilst the Quality of Service for the high priority traffic is not degraded.
\end{abstract}

Keywords:

MOEA, Integrated control plane, Optical translucent network, GMPLS, GPON.

\section{Introduction}

Increasing energy consumption has become one of main concerns for the network development. Research in energy efficient networking has considered various network domains (e.g. core, metro and access networks) and network layers. Various effective solutions have been identified, e.g. node/link sleeping during off-peak hours [1], low-power idle [2] and traffic engineering [3]. However, there are fewer works proposed to maintain a high level of Quality

*+4474037975 65, xin.chen@eecs.qmul.ac.uk 
of Service (QoS) at the same time, which is one of main concerns of operators and service providers. The techniques used in node/link sleeping or traffic engineering are usually accompanied by a certain degree of QoS compromise. In this paper, in order to maintain a high QoS whilst building an energy efficient optical network, a flexible routing mechanism is proposed using a novel integrated control platform.

The proposed novel integrated control plane structure is to improve the traditional network operations by increasing the communication efficiency across multiple network domains. The network control mechanisms today are horizontal separations between core and access networks, and vertical separations between application, transport and physical layers. These separations can lead to high operational and energy costs, low network efficiency and high latency in path provisioning. The emergence of applications with high data rate and low delay demands, i.e. HDTV and VoIP, have posed new challenges for bringing down the energy costs whilst maintaining the QoS. However, it is not an easy task to provision an end-to-end QoS for such applications with the current separated control mechanisms across domains. A more unified, intelligent and cross domain controlling mechanism is needed to meet these challenges. Under the circumstances, Software Defined Networking (SDN) [4] concepts have been considered as alternatives. One of SDN features is to decouple the control plane and data plane, which gives a possibility to build an integrated control plane across domains. A programmable SDN control plane can provide flexibility in designing new functionalities and services, which is independent of the underlying physical infrastructure. Designing such an integrated control plane using SDN concepts is discussed in details in our previous work [5]. The proposed structure operates on the top of a translucent Generalized Multi-protocol Label Switching (GMPLS) controlled optical core network, together with Optical Network Units (ONU) Management and Control Interface (OMCI) [6] controlled Gigabit Passive Optical Network (GPON) access networks. The integrated control plane enables a sharing of network information across these network domains. To support an end-to-end (from access network to core network to access network) QoS control under the integrated control plane, the traffic from the access network is differentiated and the information is exchanged between the access and core networks by assistances of the control plane. Furthermore, differentiated routing can be applied with a more flexible routing structure from a path computation function in the integrated control plane. In the case considered in our previous work [5], the tailored energy efficient rout- 
ing algorithm can be applied mainly to low priority traffic, while delay and blocking sensitive traffic is not influenced. We choose a centralized, overlay model [5] [7] to design the integrated control plane.

Within the context of centralized control, it is possible to accomplish more complex or computational heavy tasks without increasing the resource consumption. Traditionally, the most used dynamic routing algorithms, e.g.Shortest Path (SP) algorithm or Load Balancing (LB) algorithm, can only evaluate one parameter at a time. By simply applying energy efficient routing algorithms, e.g. Least Consumption (LC) algorithm [5] on the part of network traffic that is not delay or blocking sensitive, certain energy savings can be achieved without influencing other types of traffic. Such routing strategies are limited in that only one criterion (i.e. energy consumption, delay or blocking) is considered at a time. The idea presented in this work aims to improving routing techniques by utilizing the centralized control as well as a multi-objective routing mechanism. Instead of considering hop count as the only objective for the delay sensitive traffic, energy consumption is also considered as a secondary objective.

In this paper, a Multi-Objective Evolutionary Algorithm (MOEA) is used for improving the route selection in a dynamic network context. A nondominated sorting-based algorithm called Strength Pareto Evolutionary Algorithm 2 (SPEA2) [8] is used for assigning the fitness value to each candidate solution. The algorithm is tested in a dynamic simulation environment using OPNET [9]. Results from the multi-objective algorithm are compared against the results obtained from the single objective dynamic routing algorithms (SP, LB and LC algorithms [5]). Note that a certain time is required to execute the heuristic algorithm because of the complexity of the algorithm, which is a dilemma when it supports time sensitive services in the network. The proposed algorithm's execution is based on the assumptions that a suitable computational environment exists and a certain delay tolerance is acceptable when setting up an optical core network path. The results provide input into improved schemes for real world dynamic scenarios.

\section{Related Work}

Energy efficiency in optical networks has been widely considered. In [10], the authors compare energy efficiency with network topologies and architectures. The authors in [11] use power-aware provisioning methods to achieve energy savings in optical networks including grooming operations. There are 
other similar works, but few of them take QoS requirements into consideration. In order to enable a differentiated service in the core network, similar to the concept in this work, the authors in [12] explored the possibilities of using MPLS Traffic Engineering (MPLS-TE) in the core network. However, the method has limited use in an optical network environment. Similar to the integrated control plane raised in this work, a unified control plane concept has been discussed by a number of researchers [13], [14] and [15], where end-to-end QoS is the driver. Similar concepts are developed with SDN technologies where [16] and [17] proposed an unified control plane to use with cloud services and optical burst switching. However, these works do not consider the impact on energy efficiency. The Internet Engineering Task Force (IETF) Internet draft proposed in 2013 [18], has defined details for network control functions for transport SDN. The concept is similar to our proposal, with a more general and broader scope. However, there is no actual implementation discussed in the work. Evolutionary Algorithms (EA) have been widely used in solving the Routing and Wavelength Assignment problems in optical networks. In [19], the author discusses using an EA approach to solve static RWA problems, and the author in [20] uses EA in forming both single and multi-objective problems. Neither of these has tried to apply the solutions in a dynamic environment, nor designs tailored to the needs of different applications.

\section{Software Defined Integrated Control Plane Architecture}

In this section, the integrated control plane architecture is discussed. The control plane serves as a platform to support the differentiated routing mechanisms proposed in this work. Fig. 1 shows an overview of the network architecture including the information flow, which is distributed in both the core network and access network. An optical translucent mesh structure is considered for the core network, and a point to multi-point GPON structure is considered for the access network. As an overlay layer to the GMPLS and OMCI control plane, the integrated control plane interacts with both of these. The traffic type information is encapsulated using GPON Encapsulation Method (GEM)[21] labels in the GPON network part, extracted to the integrated control plane, and translated to the traffic type for the core network.

Each connection request is treated as a traffic flow. The flow table is set up and maintained in the integrated control plane, and is implemented in 


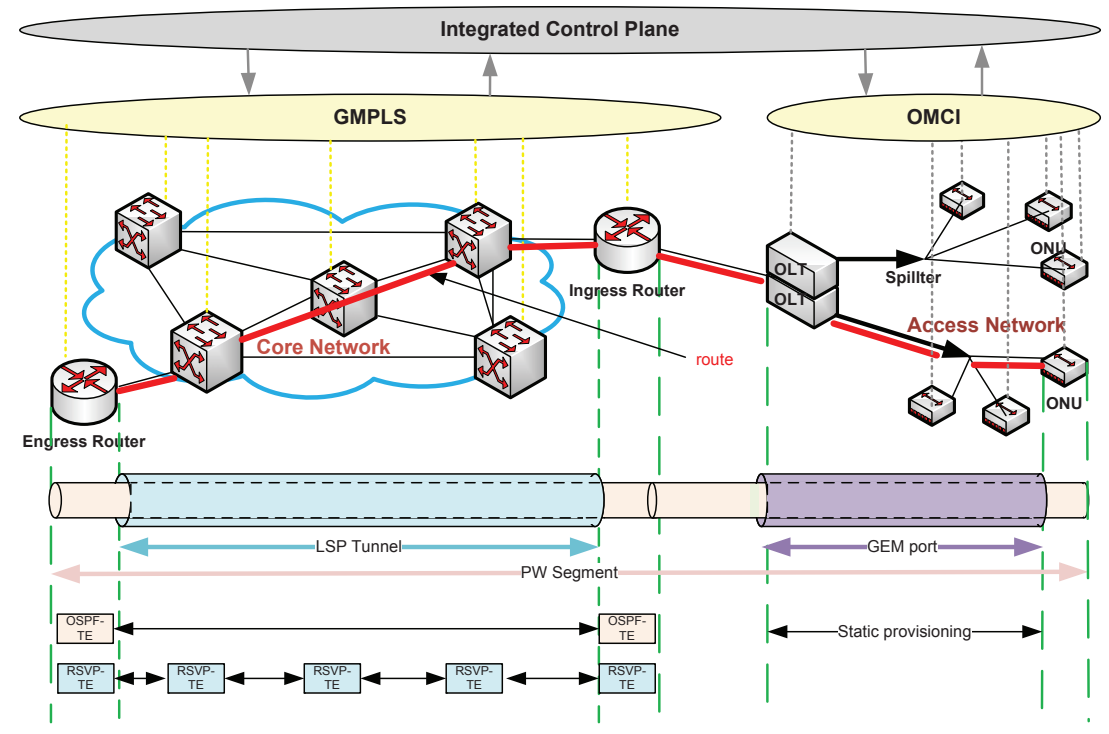

Figure 1: Network architecture considered.

a centralized manner. The information stored in the flow table can be user defined, e.g. to include traffic type and duration information. To enable a flexible routing structure, the traffic type information is needed in the flow table, together with the information necessary to define a traffic request, e.g. the required bandwidth, source and destination node address. Unlike a routing table lookup in the IP network, a flow table look up is performed only at the ingress router of the core network. If no entry is found in the flow table, a new request will be initiated by the GMPLS control plane to the integrated control plane and route calculation is performed based on the QoS requirements of the traffic flow. Once a route is obtained, the GMPLS control plane is then invoked to perform resource reservation. If the resources are successfully reserved (i.e. there exists a possible connection to support the flow) by the GMPLS control plane, the reserved route information is returned back to the integrated control plane, and the connection ID is inserted into the corresponding flow table entry. The flow setup is then complete.

As an overlay model upon both the GMPLS and the OMCI control plane, the integrated control plane is designed to perform the route computation task while maintaining an information exchange for both control planes across domains. Fig. 2 shows the interaction between the integrated control plane 
and the underlying architecture. The GMPLS control plane maintains the majority of its functionalities, but the Path Computation function is taken over by the integrated control plane. The OMCI control plane still maintains its own functionality. Inside the GMPLS controller unit, the Network Connection Controller (NCC) is responsible for setting up Path Computation Requests (PCReq), the Signaling Controller (SC) and Routing Controller (RC), implementing RSVP-TE and OSPF-TE protocols, respectively. The OMCI control plane allows multiple GEM port labels [21] provisioned between OLT and ONUs, which indicates different service/traffic types. OMCI uses static provisioning of GEM port labels. The mapping of GEM port labels from OMCI to the supported traffic class is pre-defined. The integrated control plane unit can obtain the GEM label information through the OMCI interface. Inside the integrated control plane unit, the Connection Manager (CM, shown in two parts in Fig. 2 for illustration purposes) is responsible for handling routing requests, maintaining the label mapping information and interacting with other parts of the integrated control plane. In the case presented, the QoS labels from both the access and core network parts are translated to be associated with traffic types. Routing algorithms are installed in the integrated control plane, and can be called by the path computation function. This separate space provides the possibility to execute flexible routing algorithms such as the proposed MOEA is sited. The execution of the algorithm is supported by the TE Database, which stores information collected by the GMPLS routing protocol (OSPF-TE). The PCReq is handled in the Connection Manager (CM) unit. Once the CM contacts the Path Computation unit with a PCReq, the path computation results are returned to the CM, which also contacts the Flow Processor to create a new flow entry, accordingly. Note that the wavelength reservations are still handled by GMPLS using a standard RSVP-TE path reservation process. At the access network domain, the Connection Manager is used to retrieve information through the OMCI, which includes rules for assigning GEM labels to different traffic types in the OMCI control plane. Such rules for assigning GEM labels are then used to map the traffic type information defined in other parts of the network, to enable an end-to-end QoS provisioning. 


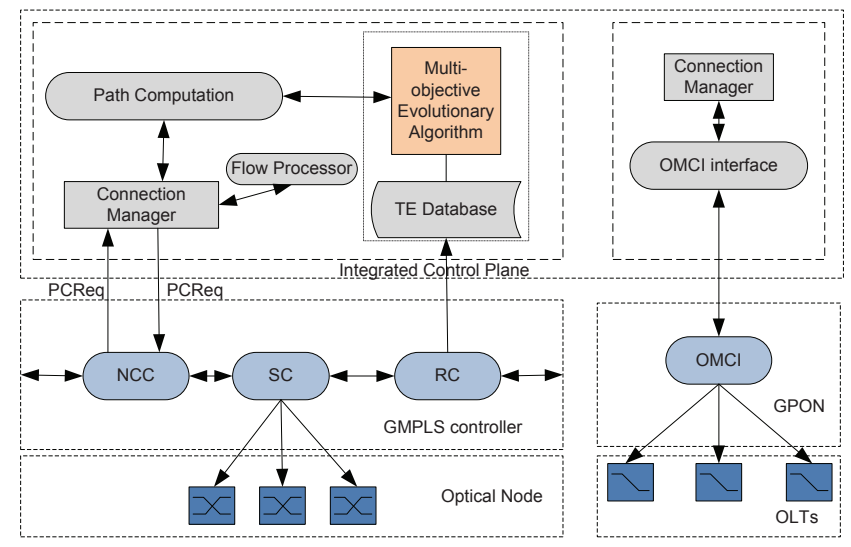

Figure 2: Interaction between the integrated control plane and the underlying structure.

\section{Multi-objective Routing Problem: Design and Implementation}

\subsection{Introduction}

An MOEA is to solve a problem involving multiple conflicting objectives using evolutionary mechanisms, such as mutation, crossover and selection. The problem search space is usually intractably large and highly complex. There is no single solution that is able to simultaneously optimize all the conflicting objectives. Therefore, the outcome of an MOEA is a set of trade-offs or compromises called Pareto optimal solutions. A solution is Pareto optimal when none of the objective values can be improved without degrading some of the other objective values. MOEAs work on a group of candidate solutions called a population. MOEAs typically start with a population generated by a random method or a predefined scheme. The population is then refined iteratively by employing two basic principles: selection and reproduction. The selection mechanism mimics the fierce competition for survival in the natural world. The fittest ones survive and have a chance to produce offspring. A reproduction mechanism, including mutation and crossover genetic operators, imitates the process of producing offspring, sharing genetic information between parents and changing genes accidentally. When MOEAs reach the stopping criterion, for example, a predefined maximum generation, MOEAs stop and a group of good solutions are obtained.

MOEA is a generic methodology and has to be customized to the particular problem. In our route selection problem, we have three types of traffic: VoIP, IPTV and web-surfing. VoIP and IPTV traffic have different pairs of 
conflicting objectives. The solutions require consideration of both objectives. Therefore, an MOEA based algorithm is applied to identify a group of good solutions. The proposed algorithm is invoked each time a connection request arrives at the ingress point.

\subsection{Problem Definition}

Given a set of random routes between the source and destination pair, the aim is to find a route with a good trade-off between the two objectives, based on the current network state and the traffic type.

\subsection{Design Parameters [5]}

1. An optical network of a graph $G=(V, E)$ where $V$ is the number of vertices and $E$ is the number of edges in the network. Optical Amplifiers (OAs) are placed on the network edges and regenerators are placed on the network node.

2. A route from node $i$ to node $j$ is denoted as $R_{i j}=\left\{a_{1}, a_{2}, a_{3}, \ldots a_{n}\right\}$, where $\left\{a_{1}, a_{2}, a_{3}, \ldots a_{n}\right\}$ is a string of integers with $n \leq V$.

3. Maximum length of each solution (route) $R=E$.

4. Maximum number of wavelengths per link is $W=24$.

5. Maximum length without the need of amplification is $\theta_{O A}=80 \mathrm{~km}$.

6. Maximum length without the need for regeneration is $\kappa_{3 R}=1000 \mathrm{~km}$.

7. Energy consumption for transporting the optical signal is $o p_{n}=0.062 n \mathrm{~J} / \mathrm{bit}$.

8. Energy consumption for add/drop and regenerations is $e l_{n}=3 n \mathrm{~J} / \mathrm{bit}$.

9. The connection requests interarrival rate and connection duration are exponentially distributed, thus the offered traffic load can be calculated in Erlangs. The mean value for connection duration is $d_{p}=6$ hours.

10. The mutation rate is 0.1 and crossover rate is 0.9

11. The initial population is $P_{0}$ and secondary population is $S_{0}$. In generation $n$, the primary population is $P_{n}$ and secondary population is $S_{n}$. The mating pool is $M_{n}$

12. The size of primiary population $P$ is $P_{\text {size }}=20$, the size of secondary population $S$ is $S_{\text {size }}=10$ and size of the mating pool $M$ is $M_{\text {size }}=5$.

13. Maximum generation number in the MOEA is $G_{\max }=100$. 


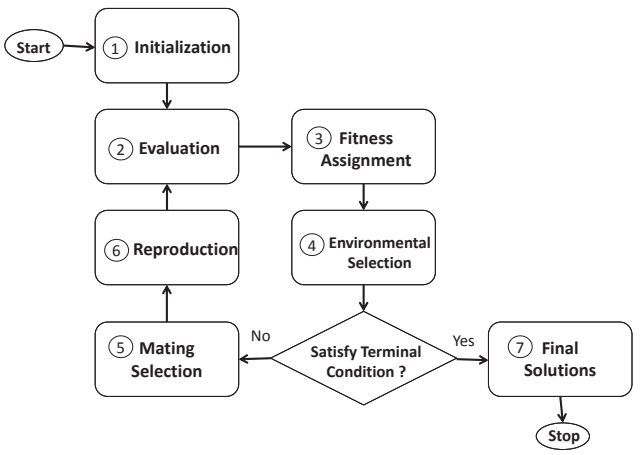

Figure 3: MOEA flowchart.

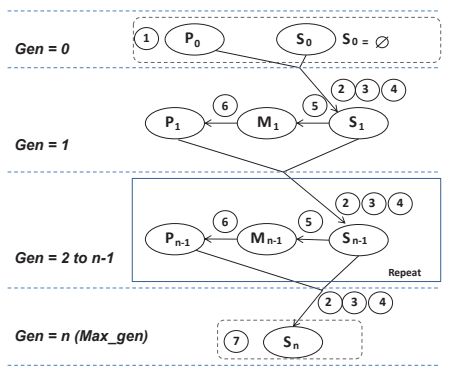

Figure 4: Evolution of three populations.

\subsection{Constraints}

1. Wavelength continuity constraint: The route from a single source to destination pair must employ the same wavelength end-to-end.

2. $a_{p} \neq a_{q}$, where $p, q \in\{1,2, \ldots, n\}$. No identical integer is allowed in an individual chromosome, i.e. no loops are allowed in the route. The maximum length of $R_{i j}$ is thus equal to $V$.

\subsection{Proposed Route Selection Algorithm}

In the proposed route selection algorithm, a candidate solution represents a selected path composed of a string of integers, where each integer identifies a node on the path, from a source to a destination node. Three populations: a primary population $P$, a secondary population $S$ and a mating pool $M$ are used in the algorithm. The primary population is a regular population which is replaced by offspring every generation. The function of secondary population is to archive nondominated solutions so no matter how many generations are iterated, the best nondominated solutions will be retained. The mating pool is used to accommodate parents that will be used to produce offspring. The size of three populations is fixed. The algorithm flowchart is shown in Fig. 3 and each step is described in detail from Section 4.5.1 to 4.5.7. The evolution of the three populations is illustrated in Fig. 4. The step number along the arrow indicates the operation(s) happen amongst populations. 


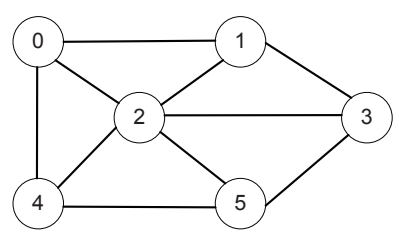

Figure 5: Search space for requests from node 0 to 5 .

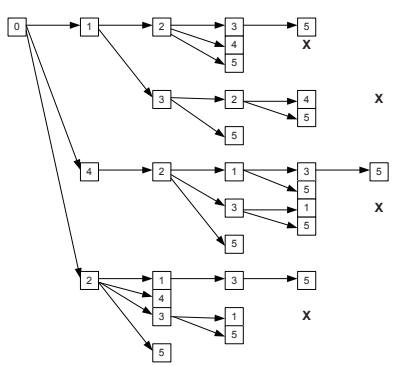

Figure 6: Possible individual representation.

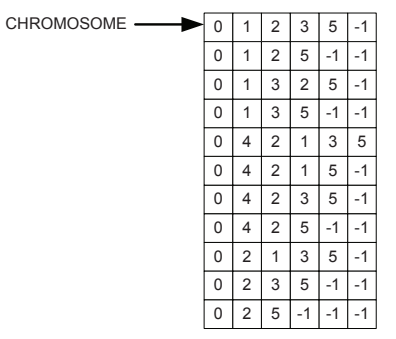

Figure 7: Example 6 node graph initial population and encoding.

\subsubsection{Initialization}

An initial primary population $P_{0}$ with $P_{\text {size }}$ randomly generated solutions is created and an empty secondary population $S_{0}$ is generated. For a given source and destination pair, each potential solution is generated according to a predefined method as follows: The source node is taken as the first node on the path, and then one of its neighbours (directly adjacent nodes) is randomly selected as the next node. The adjacent node is marked as visited and becomes the current node. If the node is not the destination node, the process repeats by randomly choosing one of its non-visited neighbouring nodes as the next node until the destination node is reached. A simple 6node-10-link topology is shown in Fig. 5. Fig. 6 shows the search space from source node 0 to destination node 5 . Fig. 7 gives the possible solutions, -1 is a null place holder..

\subsubsection{Evaluation}

The solutions in the primary population and the secondary population (if it is not empty) are evaluated by objective functions. In the route selection problem, each request has different objective functions based on its traffic type. For VoIP traffic, the objectives are to minimize both delay (objective one) and energy consumption (objective two); for IPTV traffic, the objectives are to minimize the blocking rate (objective one) and energy consumption (objective two). The objective of web-surfing traffic is to minimize the energy consumption. The objective functions are shown as follow:

1. Delay Minimization:

Let $E^{*}=E\left(R_{i j}\right)$ represent all the edges in route $R_{i j}$. A cost $c_{k}$ is assigned to each edge $k \in E^{*}$ where $c_{i}=c_{j}$. The cost of an individual 
is $C\left(R_{i j}\right)=\sum_{\forall k \in E^{*}} c_{k}$. A smaller $C\left(R_{i j}\right)$ indicates a shorter delay.

2. Blocking Minimization:

Let $w_{k}$ be the number of wavelengths used on an edge where $k \in$ $E^{*}$. The total number of wavelengths used by route $R_{i j}$ is $W\left(R_{i j}\right)=$ $\sum_{\forall k \in E^{*}} w_{k}$. It is assumed a lower blocking probability is obtained on routes with lower wavelength occupancy, then the lower cost of $W\left(R_{i j}\right)$ indicates a lower blocking rate.

3. Energy Minimization:

Let $e e_{i}$ be the energy consumption of a node, where $i \in V$. $e e_{l}$ is the energy consumption of an Optical Amplifier (OA). It is assumed the energy cost per edge equals half of the nodes cost at each end plus the link cost. Thus the energy cost per edge can be denoted as: $E E_{k}=\frac{1}{2}\left(e e_{i}+e e_{j}\right) \cdot b_{p}+\frac{l_{k} \cdot e e_{l}}{\omega_{k} \cdot \theta_{O A}} \cdot d_{p}$, where $l_{k}$ is the edge length, $b_{p}$ is the connection bandwidth and $d_{p}$ is the connection duration. $e e_{i}=o p_{n}$ when the traffic transparently passes through a node and $e e_{i}=e l_{n}$ when the traffic needs regenerations or add/drop within a node. The total energy cost of the individual is then $\sum_{\forall k \in E^{*}} E E_{k}$.

\subsubsection{Fitness Assignment}

When the objective value evaluation finishes, the primary population and the secondary population are merged into a temporary population for fitness assignment. We apply a fitness assignment as in SPEA2 [8] on the basis of Pareto dominance.

SPEA2 considers both the number of dominating and dominated solutions of each solution using two methods: dominance rank and dominance count. Firstly, dominance rank records the number of solutions by which a solution is dominated. A strength value is assigned to each solution according to the number of solutions it is dominated by. Secondly, a dominance count uses the number of solutions dominated by a certain solution to determine the fitness value. It means for each solution, the strength values of its dominators are summed up to form the fitness value. Thus, a higher value is actually less fit. A large fitness value means a solution is dominated by many solutions and conversely the fitness value of nondominated solutions is zero. Furthermore, the fitness assignment method assigns a dynamic fitness value to each solution rather than an absolute value. A solution (assume it survives over several generations) may have different fitness values in each generation depending on its relationship with the other solutions. 


\subsubsection{Environmental Selection}

Environmental selection determines which solutions from the primary and secondary populations will form the next generation secondary population. We apply environment selection to select a constant number of solutions to update the secondary population. This truncation method for forming the new secondary population is as follows: Firstly, all the nondominated solutions in the current primary population and current secondary population are copied into the next generation secondary population. Secondly, one of three actions is taken depending on the secondary population size $A$ and number of nondominated solutions $B$, namely:

1. If $A=B$, the secondary population updating finishes.

2. If $A<B$, the remaining places in the secondary population are occupied by the best dominated solutions from the current secondary population, or primary population, as necessary.

3. If $A>B$, excess nondominated solutions are eliminated from the secondary population based on their density information given by the Knearest measurement [24]. This means the solution with the minimum distance to another solution is iteratively eliminated until $A=B$. If two solutions have same distance with their nearest neighbors, the distance to the second nearest neighbor is compared. The solution with smaller distance to the second nearest neighbor is eliminated.

\subsubsection{Mating Selection}

Mating selection determines which solutions are chosen from the secondary population to form the mating pool to reproduce offspring. The selection mechanism is a tournament with replacement. Two solutions are randomly selected from the secondary population. The better solution is copied into the mating pool. The process repeats until the mating pool is full. It should be noted that no matter whether or not solutions are copied into the mating pool they remain in the secondary population subject to the constraints given in Section 4.5.4 to ensure good nondominated solutions persist between generations.

\subsubsection{Reproduction}

Offspring are generated according to two operations: crossover and mutation, controlled by the crossover rate and the mutation rate. Two solutions from the mating pool are selected randomly to be parents to produce two 


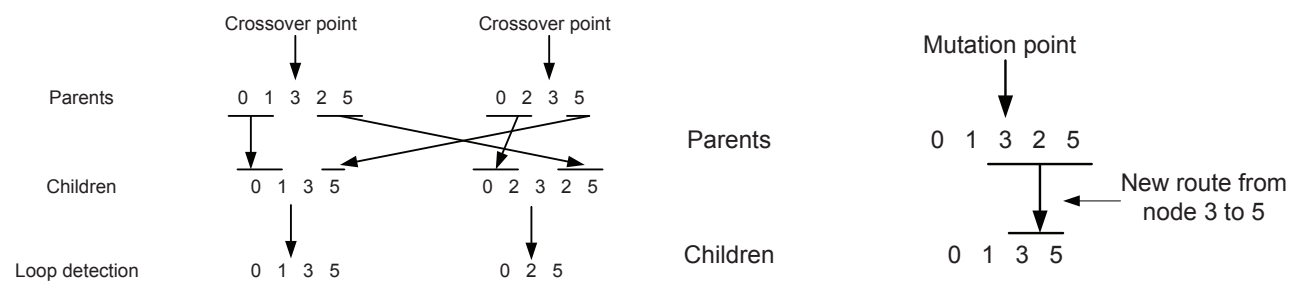

Figure 8: Example crossover. $\quad$ Figure 9: Example mutation.

children. Then the offspring are placed in the next generation primary population. The process repeats until the next generation primary population is full. The crossover operator produces offspring by exchanging gene information from the two parents. In the route selection problem, the crossover is applied to a pair of solutions that have at least one node in common (apart from the source and the destination node). A random common node is then selected as a crossover point and two parents are divided into two halves. By recombining the first part and second part of two parents, two children are reproduced. An example of a crossover operation following from the given network graph is shown in Fig. 8. A crossover point is randomly selected from a common node in both parents. The parts before and after the crossover point in both solutions are swapped and new solutions are generated. To make sure the resulting offspring are valid without any loops, additional loop detection is needed. The mutation operator is used to produce solutions based on the existing ones by altering some gene values, as shown in Fig. 8. A mutation point is randomly chosen within a target solution. A new random solution is created from the mutation point to the destination node and thus a new solution is created. When reproduction finishes, offspring become the new generation primary population. We then return to step 2 in Section 4.5.2.

\subsubsection{Final Solutions}

When the terminal condition is reached, the simulation stops. The secondary population in the final generation is the outcome. Then, the final solutions are ranked prioritizing objective one. Next, the solutions in the final population are filtered according to predefined conditions (as explained in Section 5). Finally, the best solution which satisfies the condition(s) is selected and the optical connection is established. 


\section{Results and Discussions}

The proposed structure is implemented in a simulation model using OPNET [9]. NSFNET (version year 1991, [22]) is selected as the core network topology, and each node is associated with a GPON network. The core network uses an optical translucent network architecture with Optical Amplifiers (OAs) installed at a fixed interval on each link. Regenerations are only performed when necessary. Traffic from GPON network end users are generated as a Poisson process, with an exponentially distributed connection duration. The traffic is then processed at the ingress router as Label Switched Path (LSP) requests with the requested connection duration. No wavelength conversion or grooming is employed in the core network. For simplicity, each associated GPON network is composed of three representative Optical Line Terminations (OLTs). 64 Optical Network Units (ONUs) are linked with the OLTs, with the ability to generate three types of user traffic, i.e. web-surfing, VoIP and IPTV traffic. OSPF-TE is used as the routing protocol and RSVP-TE is used as the signaling protocol in the optical translucent network. The computer environment is: Microsoft Windows XP, version 2002. Intel(R) Core(TM) 2 Duo CPU, T7500, 2.20GHz, 1.98 GB of RAM. The worst run-time complexity of the proposed MOEA algorithm is $O\left(N^{(3)}\right)\left(N=P_{\text {size }}+S_{\text {size }}\right)$, which is dominated by the truncation operator in the environmental selection [8]. An average of 0.0017 seconds is recorded for each run of the MOEA. The simulation parameters are defined as:

1. Scenario I: $\chi>3, \psi>3$;

2. Scenario II: $\chi>4, \psi>3$;

3. Scenario III: $\chi>3, \psi>4$;

4. Scenario IV: $\chi>4, \psi>4$.

Given the flexibilities in the routing calculations by the integrated control platform, in our previous work [5], three dynamic algorithms are applied on different types of traffic, i.e. LC algorithm for the web-surfing traffic, SP algorithm for the VoIP traffic and LB algorithm for the IPTV traffic. The results from single objective dynamic simulations show an improvement in energy efficiency for the low priority traffic type, i.e. web-surfing traffic, whilst the high priority traffic is not penalised in terms of blocking or delay. The proposed MOEA algorithm is designed to achieve better energy efficiency for the high priority traffic types as well by considering the energy efficiency as a secondary objective. Multiple trade-offs are given as an 
Table 1: Simulation Input Parameters.

\begin{tabular}{l|l||l|l} 
Input Parameters & Value & Input Parameters & Value \\
\hline \hline Flow setup latency & $2 \mathrm{~ms}[23]$ & MinLSInterval & $60 \mathrm{~s}$ \\
\hline $\begin{array}{l}\text { Routing table update } \\
\text { interval }\end{array}$ & 1 hour & $\begin{array}{l}\text { Wavelength Assignment } \\
\text { Algorithm }\end{array}$ & First Fit \\
\hline TE flooding interval & 3 hours & $\begin{array}{l}\text { Max LSA re- } \\
\text { transmission }\end{array}$ & 8 \\
\hline Core network link rate & $2 \mathrm{E} 8$ & Access network link rate & $1 \mathrm{E} 6$
\end{tabular}

outcome of the MOEA for each traffic request. All trade-offs/solutions are then ranked in a list based on the primary objective function values. Given the nature of the fitness function, if a solution has best performance in the primary objective, it will have the worst performance in terms of the secondary objective. Compare with the first solution, the second solution in the list have better performance on secondary objective whilst sacrificing the primary objective performance. In order to achieve better energy efficiency, the second solution is used as a preferred solution for some traffic requests. To distingush different traffic requests, we set two parameters: $\chi$ denotes the path length for VoIP traffic and $\psi$ denotes the path length for IPTV traffic. The second solution is applied on some of the traffic requests, e.g. $\chi>3$, $\psi>3$ means the second solution is applied to VoIP traffic requests with a path length of more than 3 hops, and IPTV traffic requests with a path length of more than 3 hops. Parameters are selected around 3 or 4 hops as the average hop count number for connection requests is between 3 to 4 hops under the NSFNET. In order to quantify the gain obtained from the second solution, scenarios with second solution are compared to the single objective optimization results from the previous work in terms of energy cost. The same traffic load (12, 15, 18, 24 Erlangs respectively) is assigned to each traffic type. For simplicity of implementation, no differentiated scheduling at the access point is implemented. The results are obtained with a $95 \%$ confidence interval from a 30 days runtime with 30 prime number seeds.

The total energy cost optimization result is shown in Fig. 10, where at least a $1 \%$ improvement in energy efficiency is obtained in all scenarios. Compared to the single objective case in the previous work, about $4 \%$ improvement can be be further obtained in regard to the high priority traffic. Scenario I achieves the highest savings, as the energy trade-off solutions are applied to 


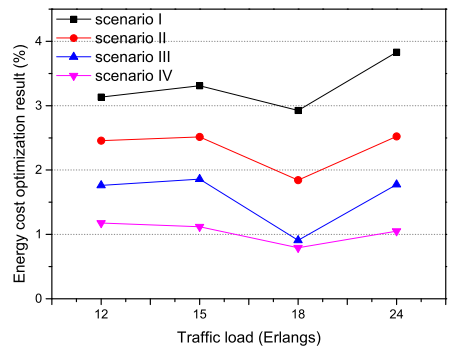

Figure 10: Total energy cost optimization degree.
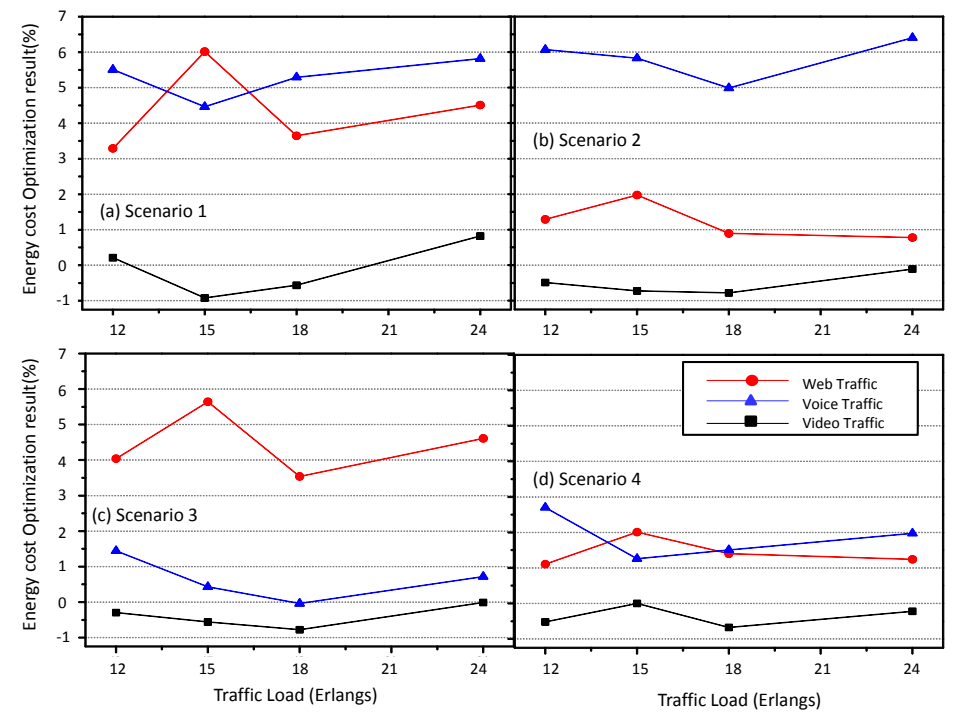

Figure 11: Energy cost optimization degree in four scenarios.

the highest number of requests. A difference in scenario II and scenario III energy savings are observed, which may be caused by the difference in the actual number of requests that is affected by the energy trade-off. As blocking minimization will naturally give a solution with a higher hop count number, the parameter of $\psi>3$ works on higher number of requests compared to that with $\chi>3$. Different routing strategies (SP,LB and LC) are applied to different types of traffic and compete for the same available resources. Thus, with increasing traffic load, the degree of optimization lessens, as shown in Fig. 10. It appears to recover for the highest traffic load case but this result excludes the large amount of blocked connection attempts. 

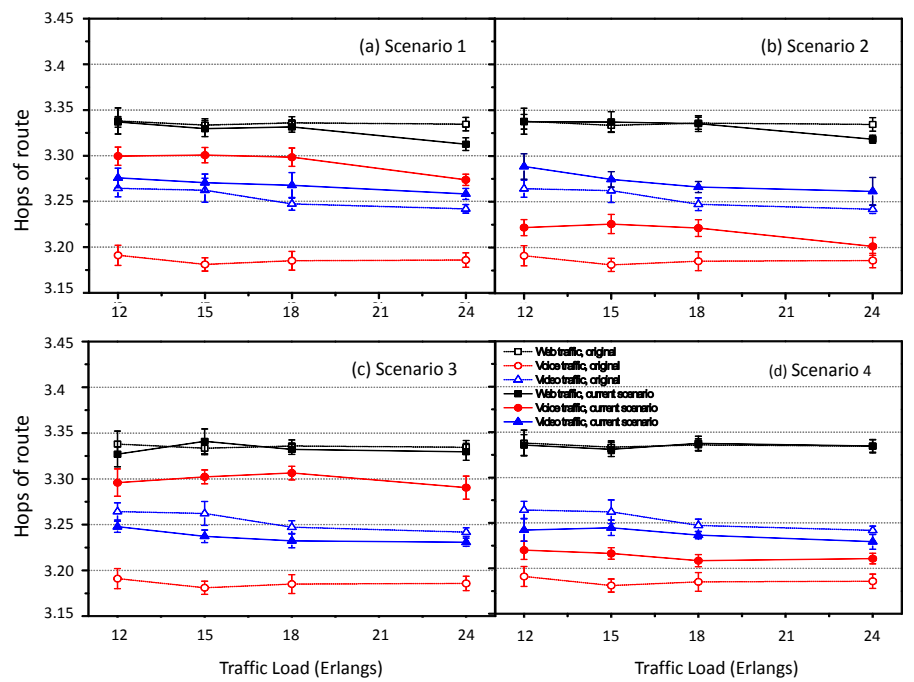

Figure 12: Hop count optimization results in four scenarios.
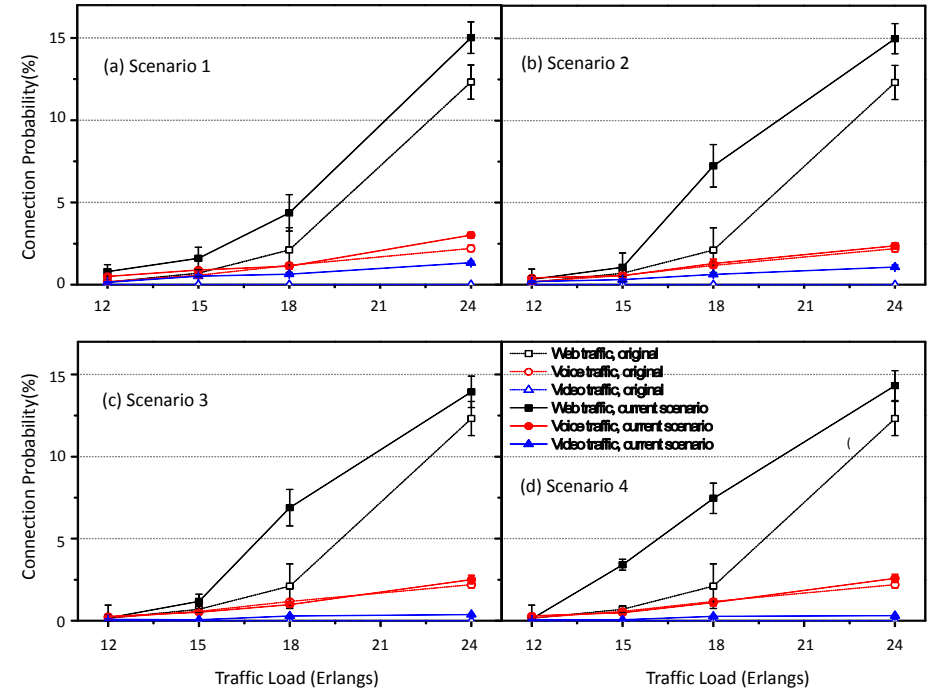

Figure 13: Connection blocking probability optimization results in four scenarios.

Compared to the dynamic single objective results from the previous work, the use of the MOEA algorithm achieves better energy efficiency for high 
priority traffic. Depending on how optimization parameters are configured in different scenarios, some degradation in delay or blocking performance is observed, as shown in Fig. 12 and Fig. 13. The change in web traffic is also shown, as a result of routing strategy changes brought about by other types of traffic. The energy saving in web traffic normally become worse as other types of traffic compete to take routes that are more energy efficient. A better energy efficiency of up to $6.5 \%$ is achieved as shown in Fig. 11. From Fig. 11 (a) to Fig. 11 (d), a clear difference in the optimization degree can be seen for both video and voice traffic. The energy improvement is obviously better when the secondary objective is considered for a higher number of requests. Fig. 11 (b) and Fig. 11 (c) show the results when one parameter is given more emphasized than the other in improving energy efficiency, where a slightly better result is observed if the emphasised parameter is used on video traffic alone, compared to Fig. 11 (a). This also increases the blocking probability for the video traffic from $0.1 \%$ to $1 \%$ with increasing offered traffic, as shown in Fig. 13 (a) and Fig. 13 (b). Similarly, a maximum of $6 \%$ energy improvement in voice traffic can results despite an approximately 0.12 hop increase, though this is still lower than the average hop count for web traffic, as shown in Fig. 12. The differences in performance are caused by the three different objectives, where delay minimization may help to obtain a shorter path, blocking minimization helps to select a less utilized route and energy minimization will help to obtain a more energy efficient route, whilst causing an increase in hop count and blocking probability. If maintaining a minimized hop count for voice traffic compared to the other types of traffic is required, the energy savings can only be reduced by up to $2 \%$. The situation is better for video traffic, as even for the maximum energy improvement case, the blocking probability is still the least among the different traffic types.

\section{Conclusion}

In this paper, a centralized integrated control plane architecture is proposed to enable a unified control across core and access domains in optical network. Such a centralized control plane not only enables a more efficient information exchange across different domains, but also gives a possibility of implementing more powerful algorithms. The work aims to deal with the conflict between energy savings and QoS maintenance. The traffic type information can be kept in the integrated control plane and used over the entire optical network domain. When employing single objective routing algorithms 
for different types of traffic, QoS can be maintained for high priority traffic and energy savings gained for low priority traffic by energy efficient routing algorithms. However, compared with such single objective dynamic routing algorithms, a multi-objective evolutionary algorithm is proposed to further optimize the energy performance across the whole network. Results show that through using the proposed MOEA algorithm, there is an opportunity to further improve the energy performance of high priority traffic without degrading QoS by taking energy saving as the secondary objective. Moreover, parameter adjustments play an essential role in the trade-off between energy savings and QoS. However, unsurprisingly, it is also observed that the performance of low priority traffic, with which energy minimization is the only objective, performance degrades when the high priority traffic puts more weights on energy minimization. This is due to more traffic connections competing for energy efficient routes across the network.

[1] Francois, F., et al., Optimization for time-driven link sleeping reconfigurations in ISP backbone networks, IEEE Network Operations and Management Symposium, 2012.

[2] Paillassa, B., et al., Performance evaluation of energy efficient policies for ethernet switches, Wireless Communications and Mobile Computing Conference, 2013.

[3] Chen,X., et al., Energy-efficient resilience in translucent optical networks with mixed regenerator placement, IEEE/OSA Journal of Optical Communications and Networking, 2013.

[4] ONF, Software-Defined Networking: The new Norm for Networks, available on line: https://www.opennetworking.org, 2013.

[5] Wang, J., et al., Design of Energy Efficient Optical Networks with Software Enabled Integrated Control Plane, IET networks, accepted for publication, 2013.

[6] TELECOMMUNICATION STANDARDIZATION SECTOR OF ITU, ONU management and control interface specification, G. 988, 2010.

[7] Liu,L., et al., Experimental Demonstration of OpenFlow/GMPLS Interworking Control Plane for IP/DWDM Multi-Layer Optical Networks, IEEE ICTON, 2012. 
[8] Zitzler, E., et al., SPEA2: Improving the Strength Pareto Evolutionary Algorithm. TIK Report 103, Computer Engineering and Networks Laboratory, ETH Zurich, Zurich, 2001.

[9] OPNET Technologies, Inc., http://www.opnet.com.

[10] Musumeci, F., et al., A Power Consumption Analysis for IP-Over-WDM Core Network Architectures, IEEE/OSA Journal of Optical Communications and Networking, 2012.

[11] Xia, M., et al., Green Provisioning for Optical WDM Networks, IEEE Journal of Selected Topics in Quantum Electronics, 2011.

[12] Tanvir, M., et al., Decreasing packet loss for QoS sensitive IP traffic in DiffServ enabled network using MPLS-TE, 2010 International Symposium in Information Technology, 2010.

[13] Orphanoudakis, T., et al., Future Internet Infrastructure Based on the Transparent Integration of Access and Core Optical Transport Networks, IEEE/OSA Journal of Optical Communications and Networking, 2009.

[14] Zhao,Y., et al., Application-oriented integrated control center (AICC) for heterogeneous optical networks, Asia Communications and Photonics Conference and Exhibition, 2011.

[15] Mohamad, A.S., et al., Development and evaluation study on GMPLS peer model to realize optical quality control, 2008 IEEE Region 10 Conference, 2008.

[16] Channegowda, M., et al., Software-defined optical networks technology and infrastructure: Enabling software-defined optical network operations, IEEE/OSA Journal of Optical Communications and Networking, 2013.

[17] Patel, A.N., et al., QoS-aware optical burst switching in OpenFlow based Software-Defined Optical Networks, International Conference on Optical Network Design and Modeling, 2013.

[18] Lee, Y., et al., Network control function virtualization for transport SDN, IETF Internet draft, 2013. 
[19] Le, V.T., et al., A Genetic Algorithm for Dynamic Routing and Wavelength Assignment in WDM Networks, Springer-Verlag Berlin Heidelberg 2004.

[20] Banerjee, N., et al., A Genetic Algorithm Approach for Solving the Routing and Wavelength Assignment Problem in WDM Networks, available at http://people.cs.umass.edu/ nilanb/papers/ICN.pdf.

[21] Li, H., et al., Multi-Segment Pseudo wires in Passive Optical Networks, RFC 6456, 2011.

[22] Available on line at http://www.nsfnet-legacy.org/about.php.

[23] Curtis, A.R., et al. DevoFlow: Scaling Flow Management for highperformance networks, SIGCOMM '11 of ACM conference, 2011.

[24] Silverman, B.W.Density estimation for statistics and data analysis, London: Chapman and Hal, 1986 